\title{
Approaches and Study Skills Inventory for Students: Comparación de las Propiedades Psicométricas entre las Versiones de Lápiz-Papel y Online en Estudiantes Universitarios
}

\author{
Approaches and Study Skills Inventory for Students: A Comparison of Psychometric \\ Properties between Paper and Pencil and Online Versions in College Students
}

\author{
Agustín Freiberg-Hoffmann ${ }^{1}$ y Agustín Romero-Medina ${ }^{2}$
}

\begin{abstract}
Resumen
Se analizan las propiedades psicométricas de la versión argentina del ASSIST y su invarianza factorial según modalidad de administración (lápiz-papel y online). Se trabajó con dos muestras de estudiantes universitarios de Argentina. Una de ellas $(n=700)$ contestó el ASSIST en formato de lápiz y papel, y la otra $(n=835)$ lo hizo de modo online. Se testeó el ajuste del modelo para ambas muestras observando adecuados índices. Luego se comparó el modelo entre los formatos de administración verificando una adecuada invarianza métrica. Asimismo, se analizó la consistencia interna de las dimensiones del instrumento para cada muestra y se las comparó entre ellas. Los resultados mostraron adecuados índices. Finalmente, no se observaron variaciones al comparar los puntajes de las dimensiones entre ambas muestras. Se discute el alcance de los resultados y sus implicancias prácticas.
\end{abstract}

Palabras clave: validez de constructo, invarianza de medición, consistencia interna, estudiantes universitarios

\begin{abstract}
The psychometric properties of the Argentinian version of the ASSIST and its factorial invariance for two administration formats (paper-pencil and online), were analyzed. Two samples were employed. In one of them, the participants $(n=700)$ completed the ASSIST in paper and pencil format, and in the other $(n=835)$, they responded online. The model fit for both samples was tested obtaining adequate indices. A factorial invariance analysis then compared the model for the two administration formats, verifying adequate metric invariance. Furthermore, the internal consistency of the instrument dimensions for each sample was analyzed and compared. Results showed good coefficients. Finally, the comparison of dimension scores between the samples did not produce variations. The results and their practical implications are discussed.
\end{abstract}

Keywords: construct validity, measurement invariance, internal consistency, college students

\footnotetext{
${ }^{1}$ Doctor en Psicología. Investigador y Profesor Asistente de Teoría y Técnica de Investigación y Diagnóstico Psicológico, Módulo 1. Universidad de Buenos Aires / Consejo Nacional de Investigaciones Científicas y Técnicas. General Juan Lavalle 2353; C1052AAA; Buenos Aires, Argentina. Tel.: 540114952 - 5481. Correo: agustinfreiberg@ gmail.com

${ }^{2}$ Doctor en Psicología. Investigador y Profesor Titular de Psicología Básica. Universidad de Murcia, Facultad de Psicología. Campus Universitario de Espinardo - 30100 Murcia, España. Correo: agustinr@um.es
} 


\section{Introducción}

Los enfoques de aprendizaje se describen a partir de la combinación entre intereses y procesos cognitivos que los estudiantes ponen en juego durante una situación de aprendizaje (Entwistle \& Peterson, 2016). El constructo contempla tres tipos de enfoques que son Profundo, Superficial y Estratégico. El Profundo incluye una motivación intrínseca y la capacidad para articular información variada. El Superficial contempla una motivación extrínseca y la habilidad para memorizar y reproducir información. El Estratégico, por su parte, se basa en una motivación hacia el logro académico y una destreza en el manejo de recursos para el estudio gestión del tiempo, organización en el estudio y respuesta a las demandas de evaluación-. Este enfoque Estratégico se combina con el Profundo y el Superficial con el fin de alcanzar buenos resultados académicos (Entwistle \& Tait, 1990; Entwistle, McCune, \& Tait, 2013).

De los tres enfoques de aprendizaje, el Profundo, es el que se asocia con mayor frecuencia con el éxito académico (Karagiannopoulou \& Entwistle, 2019), aunque sin descartar en esa asociación al enfoque Estratégico. Por esta razón es que se intenta promover ambos en los estudiantes.

El tipo de enfoque de aprendizaje que adopte un estudiante depende principalmente de tres factores que son, el contexto académico -sobre todo actividades formativas y de evaluación-, el contenido que se debe aprender y las características individuales de los estudiantes. En este sentido, se destaca que los dos últimos factores suelen permanecer relativamente estables a lo largo de un curso, mientras que el factor relacionado con el ambiente puede presentar algunas variaciones a lo largo del tiempo (Entwistle, 1991; Gálvez-Nieto, Salvo, \& TrizanoHermosila, 2018; Inzunza et al., 2018). Por esta razón, es que se atribuye mayor importancia al contexto de aprendizaje cuando se analiza el nivel de procesamiento que los alumnos adoptan durante una situación de aprendizaje.

El contexto de aprendizaje comprende además del quehacer docente, el tipo de relaciones que se establecen durante las actividades académicas. Así, por ejemplo, actividades que incluyan debates grupales y aprendizaje colaborativo que implique la puesta en juego de procesos cognitivos de selección, organización e integración de contenidos, producen en los estudiantes una mejora en el tipo de aprendizaje Profundo (Zhou, Chen, \& Chen, 2019). En este sentido, se observa que los contextos colaborativos y competitivos, donde no hay una participación activa del docente, estimulan en los estudiantes un mayor enfoque Profundo en comparación con ambientes donde el docente tiene un rol preponderante (Çolak, 2015). Se plantea así, que la participación activa de los estudiantes en las distintas etapas del proceso de aprendizaje búsqueda de información, formulación de preguntas, integración de información, síntesis de lo aprendido, reflexión sobre el contenido aprendido y discusión con pares y docentefavorecería el desarrollo del enfoque Profundo (Hasanpour-Dehkordi, 2016).

Así, si se pretende estimular en los alumnos un aprendizaje basado en la comprensión del contenido académico, sería importante adecuar el contexto de aprendizaje de modo tal que las prácticas de enseñanza y evaluación promuevan un tipo de enfoque Profundo o incluso Estratégico en sus estudiantes. La promoción de estos dos enfoques constituye un tema muy importante dado que, además de contribuir con la mejora del rendimiento académico en los estudiantes, se asocia también con el aprendizaje autorregulado y el empleo de un mayor número de estrategias de aprendizaje, estrategias metacognitivas y estrategias de afrontamiento al estrés académico, entre otras variables (De la Fuente, Sander, Kauffman, \& Yilmaz Soylu, 2020; Erdogan \& Ozdemir, 2018; McDonald, Reynolds, Bixley, \& Spronken-Smith, 2017; Ossa-Cornejo \& AedoSaravia, 2014; Zárate-Santana, Patino-Alonso, Sánchez-García, \& Galindo-Villardón, 2021).

Para poder ajustar el ambiente con el fin de promover los enfoques Profundo y Estratégico en los alumnos, previamente se vuelve necesario conocer el tipo de contexto en el cual se encuentran inmersos los estudiantes para poder planificar las acciones a adoptar. En este sentido el análisis de los enfoques de aprendizaje ofrece información -al menos indirecta- sobre cómo los estudiantes perciben el contexto de aprendizaje.

Uno de los instrumentos más difundidos en la evaluación de los enfoques de aprendizaje es el 
Approaches and Study Skills Inventory for Students (ASSIST; Tait, Entwistle, \& McCune, 1998). Se trata de un instrumento de breve administración que mediante 18 ítems permite evaluar de modo veloz los tres enfoques de aprendizaje -Profundo, Superficial, Estratégico-. El instrumento presenta evidencias de validez de constructo -análisis factorial exploratorio con método de máxima verosimilitud para la extracción de factores- y adecuada consistencia interna en sus dimensiones $(\alpha>$.70). El ASSIST dispone de adaptación al contexto universitario de Argentina (Freiberg-Hoffmann \& RomeroMedina, 2019). La versión local del instrumento, replica, en una muestra de estudiantes de Psicología la estructura trifactorial propuesta por los autores originales (Tait et al, 1998), aunque suprime a través de un análisis factorial exploratorio -con método de estimación - cuatro ítems. La estructura es posteriormente verificada mediante un análisis factorial confirmatorio -con método de estimación máxima verosimilitud robusto- con alumnos de las facultades de Psicología e Ingeniería, que informa adecuados índices de ajuste CFI (.946) y RMSEA (.049). También se verifica la invarianza factorial del modelo según la variable facultad, obtenido valores inferiores a .01 y .015 para los índices $\triangle$ CFI y $\triangle$ RMSEA respectivamente, y un $p$ valor superior a .05 para el índice Satorra-Bentler. La consistencia interna para los tres enfoques fue óptima $\left(\alpha_{\text {ordinal }}>.80\right)$. También se han reportado adecuadas evidencias de validez concurrente con otro instrumento que evalúa enfoques de aprendizaje $(p<.01)$. Resultados similares han sido informados por estudios realizados en otros países -España, Sudáfrica, Noruega-, dando cuenta de la robustez de la estructura trifactorial del instrumento (Bonsaksen, 2018; Romero-Medina et al., 2013; Simelane-Mnisi \& Mji, 2017).

El inventario se administra de manera presencial mediante formato de lápiz y papel. Esta modalidad de administración es muy efectiva cuando se pretende conocer los enfoques de aprendizaje de un solo estudiante. En cambio, cuando se quiere evaluar el constructo de modo colectivo en un grupo de alumnos la administración online parece ser una mejor alternativa.

Si bien la administración de los tests se realiza clásicamente a través del formato de lápiz y papel, actualmente la administración remota de las pruebas comienza a cobrar mayor importancia. Es así como varios investigadores evalúan en estudiantes universitarios de modo online una gran variedad de variables psicológicas tales como depresión, ansiedad, apego y autoeficacia entre otras (Alhadabi \& Karpinski, 2019; HyseniDuraku, 2017; Joeng et al., 2017).

Distintos estudios informan acerca de las ventajas que tienen los instrumentos administrados de modo online -a través de internet-, por sobre su equivalente en lápiz y papel. Se mencionan así, una reducción del efecto de la deseabilidad social, mayor honestidad en las respuestas de los evaluados y menor número de respuestas ausentes, entre otras cosas (Gnambs \& Kaspar, 2015; Kongsved, Basnov, HolmChristensen, \& Hjollund, 2007; Vésteinsdóttir et al., 2018).

Asimismo, dentro del ámbito de la investigación, la administración de instrumentos a través de internet ofrece tres ventajas. La primera remite a una mayor generalización de los resultados, ya que la herramienta puede aplicarse a sujetos de distintas zonas geográficas. La segunda, alude a una mayor voluntad en la participación de los evaluados, dado que no sufren la presión que ejerce la presencia del investigador, pudiendo decidir con libertad sobre su participación. La tercera y última ventaja se relaciona con la mayor validez ecológica de los resultados, ya que el evaluado contesta al instrumento en una situación cotidiana (Kim, Gabriel, \& Gygax, 2019).

Todas estas ventajas son a su vez características que podrían afectar a las respuestas de los evaluados, provocando diferencias en los resultados al aplicar el instrumento a través de distintos formatos. Uno de los primeros autores en advertir esta dificultad fue Cronbach (1990), quien destaca la importancia de verificar la equivalencia de las puntuaciones de un instrumento cuando el mismo se administra en formatos diferentes. Luego, la American Psychological Association, establece en 2001, a través de la Task Force on Internet Testing ciertas condiciones que deben cumplir las versiones de los instrumentos administrados a través de internet para poder ser aplicados. De esta manera se destaca la necesidad de que los instrumentos 
cumplan con los estándares clásicos en psicometría (evidencias de validez y confiabilidad), garanticen la validez de las inferencias derivadas de su aplicación y posean manuales y normas de interpretación propia (Naglieri et al., 2004).

Posteriormente, la International Test Commission (ITC, 2005) introduce la importancia de analizar la equivalencia entre las versiones del instrumento administrado en formato de lápiz y papel e internet. La ITC destaca que, se debe demostrar que ambas versiones poseen: a) índices de confiabilidad comparables, b) correlaciones entre índices de confiabilidad en niveles aceptables, c) correlaciones con otros tests en tanto criterios externos, y d) producir puntajes con medias y desvíos comparables.

Dada la relevancia que los enfoques de aprendizaje tienen a la hora de planificar estrategias de enseñanza orientadas a lograr en los estudiantes una mayor comprensión de los contenidos académicos y aprendizajes duraderos, es que se propone validar el ASSIST en su formato de administración a través de internet de modo de aportar un instrumento que permita recoger datos del constructo en un grupo amplio de estudiantes de forma más rápida y eficiente. Para esto se proponen los siguientes objetivos: 1) Analizar la equivalencia de la estructura del instrumento en ambos formatos de administración (lápiz-papel e internet), 2) Evaluar de manera progresiva la invarianza factorial del modelo entre ambos formatos de administración (lápiz- papel e internet), 3) Investigar la equivalencia entre los índices de consistencia interna de ambos formatos de administración (lápiz-papel e internet), y 4) Examinar la equivalencia entre los puntajes observados de las dimensiones entre ambos formatos de administración (lápiz-papel e internet).

\section{Método}

\section{Participantes}

Se trabajó con dos muestras de estudiantes universitarios de Buenos Aires (Argentina). Una de ellas, integrada por 700 estudiantes $(66 \%$ mujeres, 34\% varones) de entre 17 y 56 años de edad $(M=22.19 ; \quad \mathrm{DE}=4.917 ; \quad M d n=21)$ de dos facultades (79\% Psicología, 21\% Ingeniería), respondió al instrumento de modo tradicional mediante lápiz y papel. La otra muestra, conformada por 835 estudiantes $(78.7 \%$ mujeres, $21.3 \%$ varones) de entre 17 y 59 años de edad $(M=25.21 ; \quad \mathrm{DE}=6.726 ; \quad M d n=23)$ de distintas facultades $(30.9 \%$ Psicología, $13.8 \%$ Ciencias Económicas, $12.5 \%$ Filosofía y Letras, $11.9 \%$ Ciencias Sociales, 9.6\% Derecho, 7.5\% Artes Musicales y Sonoras, $5.1 \%$ Ingeniería, $5.1 \%$ Medicina, 3.6\% Ciencias Exactas y Naturales), completó el instrumento a través de internet.

\section{Instrumentos}

Approaches and Study Skills Inventory for Students (ASSIST) (Tait et al., 1998): Se aplicó la versión del instrumento adaptada a estudiantes universitarios de Argentina (Freiberg-Hoffmann \& Romero-Medina, 2019). Esta versión conserva 14 de los 18 ítems originales, debiendo los mismos ser respondidos en una escala Likert de cinco opciones. El inventario evalúa los enfoques de aprendizaje Profundo -2, 6, 10, 12, 15, 17-, Superficial -1, 4, 8, 14, 16, 18- y Estratégico -3, 5, 7, 9, 11, 13-. El instrumento presenta evidencias de validez de contenido, aparente, constructo y concurrente, como también una adecuada consistencia interna en todas sus dimensiones (alfa ordinal >.80). Se recogió adicionalmente información sociodemográfica (sexo y edad).

\section{Procedimientos}

Previo a la administración del ASSIST se solicitó a los evaluados que indiquen su conformidad y voluntad de participar en el estudio a través de un consentimiento informado. Mediante este consentimiento se comunicó a los evaluados los objetivos de la investigación y el carácter anónimo y voluntario de su participación, pudiendo dejar de responder al instrumento, si así lo desearan, en cualquier instancia de la administración.

Los casos en formato de lápiz y papel se recogieron durante el horario de clases en distintas instituciones universitarias públicas de Buenos Aires. Se contó con el aval de las instituciones universitarias y también con la debida autorización de los profesores a cargo de los cursos. 
Los casos en formato online fueron recogidos a través de grupos de estudiantes alojados en la plataforma de Facebook. Allí se invitó a los alumnos interesados en participar a ingresar a un enlace que los direccionaba a un formulario diseñado en Google Forms, donde luego de prestar su conformidad en un consentimiento informado se les permitía completar el inventario.

\section{Análisis de datos}

Para el análisis de la equivalencia de la estructura del instrumento y la invarianza métrica entre formatos de administración (lápiz-papel y online) se empleó el software LISREL 8.8. Dado que los ítems del inventario eran ordinales es que se eligió analizar los datos a partir de matrices de correlaciones policóricas y el método de estimación robust máximum likelihood (RML; Cai \& Moustaki, 2018).

Para el análisis del ajuste de la estructura interna del modelo se interpretaron los índices Normed fit index (NFI), Non-normed fit index (NNFI), Comparative fit index (CFI) y Root mean square error of approximation (RMSEA). Valores superiores a .90 en NFI, NNFI y CFI e inferiores a .08 en RMSEA son considerados aceptables (Schumacker \& Lomax, 2016). Asimismo, se interpretó la validez cruzada a través del índice ECVI (Expected cross-validation index) informado para cada muestra (lápiz-papel y online). Debiendo el valor del índice arrojado para la muestra que completó el inventario en lápizpapel ubicarse dentro del intervalo de confianza informado para la muestra online y viceversa (Rial-Boubeta, Varela-Mallou, Abalo-Piñeiro, \& Lévy-Mangin, 2006). También se analizó la equivalencia entre los parámetros estimados y las correlaciones interfactoriales a través del coeficiente $q$ de Cohen, donde valores menores a .10 indican ausencia de efecto, entre .10 y .30 corresponden a un efecto pequeño, entre .31 y .50 efecto moderado y valores superiores a .51 efecto grande (Cohen, 1988).

Para el análisis de la invarianza factorial se estimaron tres modelos anidados imponiendo de modo progresivo distintos niveles de restricción. Primero se testeó un modelo configural, sin restricciones. Luego se estimó un modelo métrico, restringiendo cargas factoriales, interceptos y varianzas de error de los ítems. Por último, se estimó un modelo estructural, restringiendo las covarianzas entre los factores (Dimitrov, 2010). Los resultados se interpretaron mediante la comparación de los índices CFI y RMSEA, donde diferencias inferiores a $.01 \mathrm{y} .015$ respectivamente son indicadores de equivalencia métrica (Davidov, Schmidt, Billiet, \& Meuleman, 2018)

Luego se estimaron los índices de consistencia interna para las tres dimensiones en ambas muestras (lápiz-papel y online). Se calcularon los coeficientes alfa ordinal y omega mediante dos programas ad hoc (Domínguez-Lara, 2018; Ventura-León \& Caycho-Rodríguez, 2017). Posteriormente se compararon los coeficientes a través del software AlphaTest (Merino \& Lautenschlager, 2003).

Por último, mediante el programa SPSS 21 (IBM Corporation, 2012) se calcularon las puntuaciones de los tres enfoques de aprendizaje (Profundo, Superficial y Estratégico) y se analizaron diferencias estadísticamente significativas entre las muestras de lápiz-papel y online mediante la prueba $t$ de Student. Se estimó adicionalmente el coeficiente $d$ de Cohen para conocer el tamaño del efecto, interpretando los valores inferiores a .20 como efecto pequeño, entre .21 y .80 efecto moderado y superior a .81 efecto grande (Cohen, 1977).

\section{Resultados}

Se realizó un análisis factorial confirmatorio para cada una de las muestras (Tabla 1). Los resultados arrojaron adecuados índices de ajuste para cada una de ellas. Todos los parámetros estimados fueron estadísticamente significativos $(p<.05)$. Al comparar los parámetros estimados y las covarianzas de ambas muestras se observaron valores inferiores a .30 en los coeficientes $q$ de Cohen. Asimismo, los índices ECVI arrojaron valores que dan cuenta de la equivalencia de la bondad de ajuste del modelo entre ambas muestras testeadas.

Luego se realizó el análisis de invarianza factorial que verificó la equivalencia métrica del modelo entre la muestra de lápiz-papel y online (Tabla 2).

A continuación, se estimó la consistencia interna para las tres dimensiones en cada muestra mediante los coeficientes alfa ordinal y omega. 
Tabla 1. Análisis factorial confirmatorio del ASSIST en muestras de lápiz-papel y online

\begin{tabular}{|c|c|c|c|c|c|c|c|}
\hline & \multicolumn{3}{|c|}{ Lápiz-papel } & \multicolumn{3}{|c|}{ Online } & \multirow{2}{*}{$\begin{array}{c}q \text { de } \\
\text { Cohen }\end{array}$} \\
\hline & Profundo & Superficial & Estratégico & Profundo & Superficial & Estratégico & \\
\hline $\begin{array}{l}\text { 2. Cuando leo un artículo o un libro intento } \\
\text { descubrir por mí mismo lo que el autor quiere } \\
\text { decir realmente. }\end{array}$ & .545 & & & .545 & & & 0 \\
\hline $\begin{array}{l}\text { 6. Con frecuencia me sorprendo a mí mismo } \\
\text { pensando en ideas de la clase cuando estoy } \\
\text { haciendo otras cosas. }\end{array}$ & .593 & & & .596 & & & .004 \\
\hline $\begin{array}{l}\text { 10. Cuando trabajo en un nuevo tema intento } \\
\text { ver por mi cuenta cómo encajan las ideas en } \\
\text { mi cabeza. }\end{array}$ & .636 & & & .681 & & & .079 \\
\hline $\begin{array}{l}\text { 12. Con frecuencia me encuentro } \\
\text { preguntándome por cosas que he oído en clase } \\
\text { o he leído en libros. }\end{array}$ & .731 & & & .736 & & & .011 \\
\hline $\begin{array}{l}\text { 15. Hay conceptos en libros de clase o en } \\
\text { artículos que con frecuencia me provocan } \\
\text { ideas propias. }\end{array}$ & .691 & & & .701 & & & .020 \\
\hline $\begin{array}{l}\text { 1. A menudo me pregunto si el trabajo que } \\
\text { estoy realizando merece realmente la pena. }\end{array}$ & & .454 & & & .435 & & .024 \\
\hline $\begin{array}{l}\text { 8. Mucho de lo que estudio tiene poco sentido: } \\
\text { son como partes o piezas inconexas. }\end{array}$ & & .517 & & & .500 & & .023 \\
\hline $\begin{array}{l}\text { 14. A menudo siento que me ahogo en } \\
\text { auténticas montañas de papeles con las que } \\
\text { tengo que lidiar. }\end{array}$ & & .598 & & & .704 & & .185 \\
\hline $\begin{array}{l}\text { 16. A menudo me preocupa si seré capaz de } \\
\text { llevar a cabo el trabajo de manera adecuada. }\end{array}$ & & .580 & & & .581 & & .001 \\
\hline $\begin{array}{l}\text { 18. Habitualmente tengo problemas para dar } \\
\text { sentido a las cosas que tengo que recordar. }\end{array}$ & & .680 & & & .623 & & .100 \\
\hline $\begin{array}{l}\text { 3. Organizo mi tiempo de estudio } \\
\text { cuidadosamente para hacer el mejor uso de él. }\end{array}$ & & & .682 & & & .762 & .168 \\
\hline $\begin{array}{l}\text { 7. Se me da bastante bien ponerme a trabajar } \\
\text { cuando tengo que hacerlo }\end{array}$ & & & .580 & & & .675 & .157 \\
\hline $\begin{array}{l}\text { 9. Me esfuerzo mucho en estudiar porque } \\
\text { estoy decidido a hacerlo bien. } \\
\text { 13. Me las arreglo para encontrar las }\end{array}$ & & & .738 & & & .762 & .054 \\
\hline $\begin{array}{l}\text { condiciones adecuadas para estudiar y realizar } \\
\text { mi trabajo fácilmente. }\end{array}$ & & & .646 & & & .596 & .082 \\
\hline \multicolumn{8}{|l|}{ Índices de ajuste } \\
\hline NFI & & .929 & & & .947 & & \\
\hline NNFI & & .937 & & & .950 & & \\
\hline CFI & & .948 & & & .959 & & \\
\hline RMSEA [IC 90\%] & & .059 [.051-.067] & & & $.061[.054-.068]$ & & \\
\hline ECVI [IC 90\%] & & $.453[.389-.528]$ & & & $.438[.378-.507]$ & & \\
\hline \multicolumn{8}{|l|}{ Correlación } \\
\hline Profundo & - & & & - & & & $\begin{array}{l}\text { Prof- } \\
\text { Sup }=.2 \\
15\end{array}$ \\
\hline Superficial & -.178 & - & & -.375 & - & & $\begin{array}{l}\text { Prof- } \\
\text { Estra }= \\
119 \\
\text { Sup- }\end{array}$ \\
\hline Estratégico & .360 & -.224 & - & .459 & -.448 & - & $\begin{array}{c}\text { Estra }=. \\
225\end{array}$ \\
\hline
\end{tabular}

Tabla 2. Análisis de invarianza factorial del ASSIST en muestras de lápiz-papel y online

\begin{tabular}{lcccc}
\hline Modelo & RMSEA & $\Delta$ RMSEA & CFI & $\Delta$ CFI \\
\hline Configural & .060 & - & .954 & \\
Métrica & .060 & .000 & .947 & .007 \\
Estructural & .060 & .000 & .946 & .008 \\
\hline
\end{tabular}

Luego se compararon los índices de ambas muestras verificándose su equivalencia (Tabla 3 ).
Por último, se calcularon los puntajes de cada enfoque para ambas muestras y se analizaron diferencias entre ambos grupos (lápiz-papel y online). Los enfoques Profundo y Estratégico no registraron diferencias significativas entre sus puntuaciones, no así el enfoque Superficial que si halló diferencias a favor de los estudiantes que contestaron al instrumento de manera online (Tabla 4). 
Tabla 3. Comparación de índices de consistencia interna del ASSIST entre muestras de lápiz-papel y online

\begin{tabular}{|c|c|c|c|c|c|c|c|}
\hline & & $\begin{array}{c}\text { Lápiz-papel } \\
\text { [IC 95\%] }\end{array}$ & $\begin{array}{c}\text { Online } \\
\text { [IC 95\%] }\end{array}$ & $X^{2}$ & $d f$ & $p$ & $q$ \\
\hline \multirow[t]{2}{*}{ Profundo } & $\mathrm{Alfa}_{\mathrm{o}}$ & .774 [.746-.799] & $.786[.762-.808]$ & .377 & 1 & .538 & .030 \\
\hline & Omega & $.777[.750-.802]$ & $.788[.764-.810]$ & .324 & 1 & .568 & .029 \\
\hline \multirow[t]{2}{*}{ Superficial } & $\mathrm{Alfa}_{\mathrm{o}}$ & $.700[.663-.734]$ & $.702[.669-.733]$ & .005 & 1 & .940 & .000 \\
\hline & Omega & .704 [.668-.737] & .708 [.675-.738] & .023 & 1 & .878 & .001 \\
\hline \multirow[t]{2}{*}{ Estratégico } & $\mathrm{Alfa}_{\mathrm{o}}$ & .755 [.724-.783] & $.791[.767-.813]$ & 2.889 & 1 & .089 & .090 \\
\hline & Omega & $.758[.727-.786]$ & $.794[.770-.816]$ & 2.967 & 1 & .085 & .091 \\
\hline
\end{tabular}

Tabla 4. ASSIST. Análisis de diferencias en los puntajes observados entre muestras de lápiz-papel y online

\begin{tabular}{|c|c|c|c|c|c|}
\hline \multicolumn{3}{|l|}{ Administración } & Profundo & Superficial & Estratégico \\
\hline \multicolumn{2}{|l|}{ Papel-lápiz } & $\begin{array}{c}M \\
\mathrm{DE}\end{array}$ & $\begin{array}{l}17.305 \\
3.556\end{array}$ & $\begin{array}{l}13.357 \\
3.467\end{array}$ & 14.500 \\
\hline \multirow{2}{*}{\multicolumn{2}{|c|}{ Online }} & $M$ & 17.619 & 13.964 & 14.382 \\
\hline & & DE & 3.652 & 3.699 & 3.308 \\
\hline \multirow[t]{5}{*}{ Levene } & & $F$ & 1.646 & 3.311 & 2.530 \\
\hline & & $p$ & .200 & .069 & .112 \\
\hline & $t$ & & -1.695 & -3.294 & .715 \\
\hline & $p$ & & . 090 & .001 & .475 \\
\hline & $d$ & & .086 & .170 & .037 \\
\hline
\end{tabular}

\section{Discusión}

La presente investigación analiza las propiedades psicométricas de la versión online del ASSIST y su equivalencia métrica con respecto al formato de lápiz y papel. Para ello, se efectúa en primer lugar, un análisis factorial confirmatorio que verifica un adecuado ajuste del modelo en ambas muestras (online y lápiz-papel). Asimismo, al comparar los parámetros y covarianzas obtenidos en cada modelo se observa una muy baja variación entre sus valores, lo que es favorable a la hipótesis de equivalencia entre ellos. Además, el ajuste entre los modelos tampoco parece variar de modo significativo dada la similitud que registran los índices de validación cruzada. Los resultados obtenidos para ambas modalidades de administración, son a su vez similares tanto a los reportados en el estudio de adaptación local del instrumento (FreibergHoffmann \& Romero-Medina, 2019), como los informados en trabajos realizados en otros países (Bonsaksen, 2018; Romero-Medina et al., 2013; Simelane-Mnisi \& Mji, 2017). Tanto el ajuste global del modelo, como los parámetros y covarianzas estimadas, no presentan variaciones en sus valores que merezcan ser mencionadas.

En segundo término, se corrobora la equivalencia métrica del modelo entre ambas formas de administración (lápiz-papel y online), mediante un análisis de invarianza factorial. Este resultado garantiza la imparcialidad de las medias factoriales, pudiéndoselas comparar (Nolte \& Elsworth, 2014).

Como tercer paso, se examina la consistencia interna de los tres enfoques para cada modalidad de administración registrando en todos los casos adecuados índices (>.70). Adicionalmente, los coeficientes obtenidos para cada versión son comparados verificando que no hay variación estadísticamente significativa entre ellos ( $p>.05)$. Si se comparan los índices de consistencia interna aquí reportados con los informados por el estudio de adaptación local del instrumento (FreibergHoffmann \& Romero-Medina, 2019), se observa una diferencia a favor del estudio original con valores superiores a .80 . Cabe destacar que los índices alfas informados en ese estudio han sido estimados a partir del análisis factorial exploratorio, el cual se realizó con una muestra homogénea compuesta solo por estudiantes de Psicología. Si se calcula, para ese estudio, el índice omega a partir de los parámetros estimados en el análisis factorial confirmatorio, efectuado con una muestra más heterogénea compuesta por estudiantes de Psicología e Ingeniería, se observa que los valores descienden notablemente $\left(\omega_{\text {Profundo }}=.725, \quad \omega_{\text {Superficial }}=.630, \quad \omega_{\text {Estratégico }}=.728\right)$ ubicándose cerca de los arrojados en el presente trabajo. De esta manera es posible observar que 
los índices de consistencia interna obtenidos en el presente trabajo son similares a los que podría haber reportado el estudio local previo si los hubiese estimado a partir del análisis confirmatorio.

En cuarto y último lugar, se estudian las puntuaciones observadas de los enfoques entre ambas formas de administración (lápiz-papel y online), verificando que los enfoques Profundo y Estratégico no presentan diferencias significativas. Por el contrario, sí se registra diferencia significativa en el enfoque Superficial, aunque la misma presenta un tamaño del efecto pequeño. Por este motivo, esta diferencia no reviste relevancia sobre todo en tareas de rastrillaje.

Como se puede observar, la información expuesta en el presente estudio permite concluir que las formas de administración del ASSIST a través de lápiz y papel, y online, son equivalentes. De esta manera, ambas modalidades pueden aplicarse indistintamente a estudiantes universitarios para evaluar sus enfoques de aprendizaje.

El funcionamiento invariante del instrumento podría explicarse a partir de distintos factores. Primero, el contenido de los ítems que integran el ASSIST no se encuentra asociado a situaciones específicas vinculadas al formato de administración. Por el contrario, indagan conductas de los estudiantes que llevan adelante para aprender los contenidos académicos sin importar la situación de aprendizaje. Segundo, los reactivos parecerían no estar influidos por el sesgo de respuesta vinculado al efecto de la deseabilidad social que podría verse minimizado en la administración online. Si bien otro estudio ha verificado un bajo efecto de la deseabilidad social sobre los enfoques de aprendizaje (Watkins, 1996), este parecería no incidir de manera significativa en las respuestas que los evaluados dan al instrumento en ambos formatos de administración. No obstante, esta hipótesis debería ser testeada en un futuro trabajo en el que se incluya una escala de deseabilidad social en conjunto con el ASSIST. En tercer y último lugar, debe mencionarse que el carácter breve del instrumento pudo haber contribuido con la disminución de respuestas azarosas de los evaluados producidas por efecto de la fatiga. Esto no es un detalle menor, ya que los estudiantes suelen producir mayores respuestas al azar por efecto de la fatiga durante el formato de administración online. Esto se debe a las constantes invitaciones que reciben los alumnos, en la actualidad, de distintos sectores académicos -investigadores, profesionales de la educación- y no académicos -calificación de servicios y sitios web- para completar encuestas online (Van Mol, 2016).

Existen algunas limitaciones que deben mencionarse. Primero, no se ha testeado la equivalencia del instrumento entre ambas modalidades de administración a través de estudios correlacionales que incluyan criterios externos, tal como ha sido recomendado por la International Test Commission (2005). En segundo lugar, no se ha comparado la estabilidad temporal de las puntuaciones entre ambos formatos de administración mediante una prueba test-retest. Este análisis aportaría información útil al momento de evaluar el efecto de posibles intervenciones entre una primera y una segunda administración de la prueba. En tercer y último término, los estudiantes que participaron del estudio eran de Buenos Aires, lo que afecta la posibilidad de generalizar los resultados a la totalidad del territorio argentino. Estas limitaciones serán abordadas en futuros trabajos que profundicen los estudios correspondientes al análisis de la equivalencia del instrumento en las distintas modalidades de administración.

Para finalizar, es importante destacar la necesidad de conocer tanto las características cognitivas y emocionales del alumnado, como los factores sociales y educativos que afectan a las instituciones, con el fin de mejorar la calidad de los aprendizajes (American Psychological Association, 2000). En este sentido, las pruebas psicológicas aplicadas al ámbito educativo poseen importantes implicancias para los estudiantes, tanto en el plano grupal, como individual. A nivel grupal, la evaluación a gran escala de las habilidades cognitivas, intereses, emociones y actitudes, entre otras variables, posibilita la planificación de intervenciones para resolver problemas actuales o futuros. A nivel individual, los instrumentos de evaluación psicológica permiten describir las fortalezas y debilidades académicas de los estudiantes, y también 
identificar los enfoques educativos a partir de las características individuales (American Psychological Association, 2000). Es en este sentido que la versión online del ASSIST tiene un papel importante, fundamentalmente en la evaluación grupal de los estudiantes acerca del modo en que perciben el contexto educativo. De esta manera se espera que la información que el instrumento arroje pueda ser empleada por los docentes con el fin de modificar sus metodologías de enseñanza y evaluación. Estos cambios incluyen, además, el trabajo docente en la modificación del tipo de relaciones que se establecen dentro del contexto de aprendizaje. Así, pueden ponerse en marcha modelos de aula invertida, donde los estudiantes cobren mayor protagonismo colaborando con el proceso de aprendizaje de sus pares. También se puede estimular el debate grupal de distintos temas en clases, donde los estudiantes deban poner en juego procesos cognitivos de selección, organización e integración de contenidos (Zhou, Chen, \& Chen, 2019). A su vez se pueden planificar actividades de tipo colaborativas o bien competitivas, donde el docente no participe en la resolución de problemas (Çolak, 2015). Tareas de esta naturaleza deben ser las rectoras de los cambios que deben efectuarse en el contexto de enseñanzaaprendizaje con el fin último de estimular en los alumnos un aprendizaje Profundo o Estratégico siempre en mayor medida que los Superficialesde los contenidos académicos.

\section{Referencias}

Alhadabi, A., \& Karpinski, A. C. (2019). Grit, self-efficacy, achievement orientation goals, and academic performance in university students. International Journal of Adolescence and Youth, 25(1), 519-535. doi:10.1080/02673843.2019.1679202

American Psychological Association. (2000). Report of the task force on test user qualifications. Recuperado de https://www.apa.org/science/programs/testing/ qualifications.pdf

Bonsaksen, T. (2018). Psychometric properties of the Short ASSIST Scales. Uniped, 41(02), 164-181. doi:10.18261/issn.1893-8981-2018-02-07

Cai, L., \& Moustaki, I. (2018). Estimation methods in latent variable models for categorical outcome variables. In P. Irwin, T. Booth \& D. Hughes (eds.), The Willey handbook of psychometric testing: A multidisciplinary reference on survey, scale and test development. (pp 252-277). Willey.

Cohen, J. (1977). Statistical power analysis for the behavioral science. Academic Press.

Cohen, J. (1988). Statistical Power Analysis for the Behavioral Sciences (2nd ed). Erlbaum.

Çolak, E. (2015). The effect of cooperative learning on the learning approaches of students with different learning styles. Eurasian Journal of Educational Research, 59, 17-34. doi:10.14689/ejer.2015.59.2

Cronbach, L. J. (1990). Essentials of psychological testing. (5th ed.). HarperCollins.

Davidov, E., Schmidt, P., Billiet, J., \& Meuleman, B. (2018). Cross-cultural analysis. Methods and applications. Routledge.

De la Fuente, J., Sander, P., Kauffman, D. F., \& Yilmaz Soylu, M. (2020). Differential effects of self- vs. external-regulation on learning approaches, academic achievement, and satisfaction in undergraduate students. Frontiers in Psychology, 11. doi:10.3389/fpsyg.2020.543884

Dimitrov, D. M. (2010). Testing for factorial invariance in the context of construct validation. Measurement and Evaluation in Counseling and Development, 43(2), 121-149. doi:10.1177/0748175610373459

Domínguez-Lara, S. (2018). Fiabilidad y alfa ordinal. Actas Urológicas Españolas, 42(2), 140-141. doi:10.1016/j.acuro.2017.07.002

Entwistle, N. (1991). Approaches to learning and perception of the learning environment. Higher Education, 22, 201-204. doi:10.1007/BF00132287

Entwistle, N., \& Peterson, E. (2016). Learning styles and approaches to studying. The curated Reference collection in neuroscience and biobehavioral psychology. doi:10.1016/B978-0-12-809324-5.05623-6

Entwistle, N., \& Tait, H. (1990). Approaches to learning, evaluations of teaching, and preferences for contrasting academic 
environments. Higher Education, 19(2), 169194. doi:10.1007/bf00137106

Entwistle, N., McCune, V., \& Tait, H. (2013). Approaches and Study Skills Inventory for Students (ASSIST). Recuperado de https://www.researchgate.net/publication/503 90092_Approaches_to_learning_and_studyin g_inventory_ASSIST_3rd_edition

Erdogan, T., \& Ozdemir, E. (2018). An investigation of learning approaches and language learning strategies: Are they related? European Journal of Education Studies, 4(11), 91-107. doi:10.5281/zenodo.1320506

Freiberg-Hoffman, A., \& Romero-Medina, A. (2019). Validación del Approaches and Study Skills Inventory for Students (ASSIST) en universitarios de Buenos Aires, Argentina. Acción Psicológica, 16(2), 116. doi:10.5944/ap.16.2.23042

Gálvez-Nieto, J. L., Salvo, S., \& TrizanoHermosila, Í. (2018). Equivalencia factorial de la escala de actitudes hacia la autoridad institucional en adolescentes (AAI-A) chilenos y colombianos. Revista Iberoamericana de Diagnóstico y Evaluación - e Avaliação Psicológica, 46(1), 109-119. doi:10.21865/ridep46.1.08

Gnambs, T., \& Kaspar, K. (2015). Disclosure of sensitive behaviors across self-administered survey modes: A meta-analysis. Behavior Research Methods, 47, 1237-1259. doi:10.3758/s13428-014-0533-4

Hasanpour-Dehkordi, A. (2016). The efficacy of three learning methods collaborative, contextbased learning and traditional, on learning, attitude and behavior of undergraduate nursing students: Integrating theory and practice. Journal of Clinical and Diagnostic Research, 10(4), 1-4. doi:10.7860/jcdr/2016/18091.7578

Hyseni-Duraku, Z. (2017). Factors influencing test anxiety among university students. The European Journal of Social and Behavioural Sciences, 1(1), 2325-2334. doi:10.15405/ejsbs.206

IBM Corporation (2012). IBM SPSS Statistics (21). Recuperado de http://www01.ibm.com/software/es/analytics/spss/

International Test Commission (2005). ITC guidelines on computer-based and internet delivered testing. Recuperado de https://www.intestcom.org/files/guideline_co mputer_based_testing.pdf

Inzunza, B., Pérez, C., Márquez, C., Ortiz, L., Marcellini, S., \& Duk, S. (2018). Estructura factorial y confiabilidad del Cuestionario de Motivación y Estrategias de Aprendizaje, MSLQ, en estudiantes universitarios chilenos de primer año. Revista Iberoamericana de Diagnóstico y Evaluación - e Avaliação Psicológica, 47(2), 21-35. doi:10.21865/ridep47.2.02

Joeng, J. R., Turner, S. L., Kim, E. Y., Choi, S. A., Kim, J. K., \& Lee, Y. J. (2017). Data for Korean college students' anxious and avoidant attachment, self-compassion, anxiety and depression. Data in Brief, 13, 316-319. doi:10.1016/j.dib.2017.06.006

Karagiannopoulou, E., \& Entwistle, N. (2019). Students' learning characteristics, perceptions of small-group university teaching, and understanding through a "meeting of minds." Frontiers in Psychology, 10. doi:10.3389/fpsyg.2019.00444

Kim, J., Gabriel, U., \& Gygax, P. (2019). Testing the effectiveness of the Internet-based instrument PsyToolkit: A comparison between web-based (PsyToolkit) and lab-based (EPrime 3.0) measurements of response choice and response time in a complex psycholinguistic task. PLOS ONE, 14(9), e0221802. doi:10.1371/journal.pone.0221802

Kongsved, S. M., Basnov, M., Holm-Christensen, K., \& Hjollund, N. H. (2007). Response rate and completeness of questionnaires: a randomized study of internet versus paperand-pencil versions. Journal of Medical Internet Research, 9(3), e25. doi:10.2196/jmir.9.3.e25

McDonald ,F., Reynolds, J., Bixley, A., \& Spronken-Smith, R. (2017). Changes in approaches to learning over three years of University undergraduate study. Teaching \& Learning Inquiry, 5(2). doi:10.20343/teachlearninqu.5.2.6

Merino, C., \& Lautenschlager, G. J. (2003). Comparación estadística de la confiabilidad alfa de Cronbach: aplicaciones en la medición educacional y psicológica. Revista de 
Psicología de la Universidad de Chile, 12(2), 129-139. doi:10.5354/0719-0581.2012.17668

Naglieri, J. A., Drasgow, F., Schmit, M., Handler, L., Prifitera, A., Margolis, A., \& Velasquez, R. (2004). Psychological testing on the Internet: New problems, old issues. American Psychologist, 59(3), 150-162. doi:10.1037/0003-066x.59.3.150

Nolte, S., \& Elsworth, G. R. (2014). Factorial Invariance. Encyclopedia of quality of life and well-being research. Springer. doi:10.1007/978-94-007-0753-5_983

Ossa-Cornejo, C., \& Aedo-Saravia, J. (2014). Enfoques de aprendizaje, autodeterminación y estrategias metacognitivas en estudiantes de pedagogía de una universidad chilena. Ciencias Psicológicas, 8(1), 79-88.

Rial-Boubeta, A., Varela-Mallou, J., AbaloPiñeiro, J., \& Lévy-Mangin, J. P. (2006). El análisis factorial confirmatorio. In: J. P. LévyMangin \& J. Varela-Mallou (Eds.), Modelización con estructuras de covarianzas en ciencias sociales (pp. 119-154). Netbiblo.

Romero-Medina, A., Hidalgo-Montesinos, M., González-Javier, F., Carrillo-Verdejo, E., Pedraja, M., García-Sevilla, J., \& PérezSánchez, M. (2013). Enfoques de aprendizaje en estudiantes universitarios: Comparación de resultados con los cuestionarios ASSIST y RSPQ-2F. Revista de Investigación Educativa, 31(2), 375-391.

Schumacker, R., \& Lomax, R. (2016). A beginner's guide to structural equation modeling. Routledge.

Simelane-Mnisi, S., \& Mji, A. (2017). Establishing the reliability and validity of the ASSIST questionnaire: A South African sample perspective. Electronic Journal of Research in Educational Psychology, 15(1), 201-223. doi:10.14204/ejrep.41.16028

Tait, H., Entwistle, N. J., \& McCune, V. (1998). ASSIST: A re-conceptualization of the Approaches to Studying Inventory. In C. Rust (Ed.), Improving students as learners. Oxford Brookes University.

Van Mol, C. (2016). Improving web survey efficiency: The impact of an extra reminder and reminder content on web survey response. International Journal of Social Research Methodology, 20(4), 317-327. doi:10.1080/13645579.2016.1185255

Ventura-León, J. L., \& Caycho-Rodríguez, T. (2017). El coeficiente Omega: Un método alternativo para la estimación de la confiabilidad. Revista Latinoamericana de Ciencias Sociales, Niñez y Juventud, 15(1), 625-627. Recuperado de https://www.redalyc.org/pdf/773/7734962703 9.pdf

Vésteinsdóttir, V., Joinson, A., Reips, U.-D., Danielsdottir, H. B., Thorarinsdottir, E. A., \& Thorsdottir, F. (2018). Questions on honest responding. Behavior Research Methods, 51(2), 811-825. doi:10.3758/s13428-018-1121-9

Watkins, D. (1996). The influence of social desirability on learning process questionnaires: A neglected possibility? Contemporary Educational Psychology, 21(1), 80-82. doi:10.1006/ceps.1996.0006

Zárate-Santana, Z.-J., Patino-Alonso, M.-C., Sánchez-García, A.-B., \& Galindo-Villardón, P. (2021). Learning approaches and coping with academic stress for sustainability teaching: Connections through canonical correspondence analysis. Sustainability, 13(2), 852. doi:10.3390/su13020852

Zhou, X., Chen, L. H., \& Chen, C. L. (2019). Collaborative learning by teaching: A pedagogy between learner-centered and learner-driven. Sustainability, 11(4), 1174. doi:10.3390/su11041174 